\title{
Icing treatment in rats with crush syndrome can improve survival through reduction of potassium concentration and mitochondrial function disorder effect
}

\author{
ISAMU MURATA $^{1}$, MAYUKI IMANARI ${ }^{1}$, MARISE KOMIYA ${ }^{1}$, \\ JUN KOBAYASHI $^{2}$, YUTAKA INOUE ${ }^{1}$ and IKUO KANAMOTO ${ }^{1}$ \\ ${ }^{1}$ Laboratory of Drug Safety Management; ${ }^{2}$ Division of Pathophysiology, Department of Clinical Dietetics and Human Nutrition, \\ Faculty of Pharmacy and Pharmaceutical Science, Josai University, Sakado, Saitama 350-0295, Japan
}

Received January 7, 2019; Accepted May 8, 2019

DOI: $10.3892 /$ etm.2019.8230

\begin{abstract}
Crush syndrome (CS), a serious medical condition, which is characterized by damage to myocytes due to pressure and is associated with high mortality, even when patients receive fluid therapy. Icing therapy over the affected muscle has been reported to be effective in improving mitochondrial dysfunction and inflammation. These effects are thought to be secondary to improvements in the leakage of potassium and myoglobin from the damaged myocytes in the early stages of disease. However, their effects on the various symptoms of CS are unclear. It was hypothesized that treatment with icing will inhibit the influence of potassium by vasoconstriction, exert anti-inflammatory effects in the affected myocytes and improve mitochondrial function The CS model constructed by subjecting anesthetized rats to bilateral hindlimb compression with a rubber tourniquet for $5 \mathrm{~h}$. The rats were then randomly divided into six groups: i) Sham; ii) CS without treatment (CS); iii) and iv) icing for
\end{abstract}

Correspondence to: Dr Isamu Murata, Laboratory of Drug Safety Management, Faculty of Pharmacy and Pharmaceutical Science, Josai University, Keyakidai 1-1, Sakado, Saitama 350-0295, Japan

E-mail: ismurata@josai.ac.jp

Abbreviations: CS, crush syndrome; CI-30, icing for 30 min over the entire hindlimb on CS rats; CI-180, icing for 180 min over the entire hindlimb on CS rats; CLI-30, local icing for $30 \mathrm{~min}$ over the affected area on CS rats; CLI-180, local icing for 180 min over the affected area on CS rats; AKI, acute kidney injury; IRI, ischemia reperfusion injury; $\mathrm{K}^{+}$, plasma potassium; CPK, creatine phosphokinase; MBP, mean blood pressure; HR, heart rate; $\mathrm{BT}$, body temperature; Hc, hematocrit; BE, base excess; AG, anion gap; BPF, Blood perfusion; ROD, reactive oxygen species; HMGB1, high mobility group box 1 ; IL-6, interleukin-6; IL-10, interleukin-10; TBARS, thiobarbituric acid reactive substances; MPO, myeloperoxidase; cyt $c$, cytochrome $c$; SEM:, standard error of mean; PTP, permeability transition pore.

Key words: crush syndrome, icing therapy, mitochondria, anti-inflammation
30 or 180 min over the entire hindlimb on CS rats (CI-30 and -180), respectively; and v) and vi) local icing for 30 or 180 min over the affected area on CS rats (CLI-30 and -180), respectively. Under continuous monitoring and recording of arterial blood pressures, blood and tissue samples were collected for biochemical analyses at designated time points prior to and following reperfusion. The survival rate, vital signs, and blood gas parameters in the CS group were lethal compared with the sham group. These were also improved in the CI-30 and CLI-30 groups compared with the CS group; however, they worsened in the CI-180 and CLI-180 groups due to hypothermia. The CI-30 and CLI-30 groups demonstrated tendencies of improvements compared with the CS group. Systemic inflammation and mitochondria dysfunction had improved in these groups compared with the CS group. We suggest icing therapy to temporarily prolong the viability after crush injury. Its effectiveness can be improved by combining it with other infusion therapies.

\section{Introduction}

Crush syndrome (CS) is a systemic dysfunction resulting from crush injuries and muscle compression. It is characterized by an unpredictable clinical course and high mortality due to cardiac arrest, shock, acute kidney injury (AKI), and inflammatory disorders (1-3). Several human and animal studies have included pathological analyses of CS. In disaster management, massive fluid resuscitation is the recommended initial management to prevent death due to cardiac arrest and AKI. However, CS is known as rescue death because approximately $20 \%$ of deaths occur shortly after extrication (4), and at a hospital, these patients are at an additional risk of systemic inflammatory response syndrome and multi organ dysfunction syndrome secondary to ischemia reperfusion due to the recommended therapy. We previously reported that in a CS model involving rats (5), they died due to cardiac failure following hyperkalemia; therefore, the outcomes in patients with CS might be improved by improving the condition of the muscles involved. Furthermore, improvements in the muscles are reported to be due to the anti-inflammatory and anti-oxidative effects that follow prevention of mitochondrial dysfunction (6). 
Cryotherapy involves the cooling via ice packs or similar methods of the skin overlying the respective muscles in order to temporarily reduce muscle temperature, induce vasoconstriction, and inhibit pain sensation. The potential benefits of cryotherapy in the healing of the muscle following various trauma-induced damages are controversial (7). In cryotherapy, icing technique is general used in the emergency treatment of trauma in addition to rehabilitation and surgery (8). Furthermore, low tissue temperature is the most powerful intervention towards limiting ischemia reperfusion injury (IRI), which may subsequently prevent mitochondrial damage and, therefore, reduce the severity of IRI (9). Basic animal studies have proved the positive effect of icing treatment over contusion injury (10) and crush injury in rats (11) in the early stages of attenuated inflammation (during migration of inflammatory cells); however, these effects were not sufficient to prevent effective muscle regeneration at later stages. Furthermore, such studies did not investigate and clarify the efficacy of icing intervention in improving survival, electrolyte balance, vital signs, and inflammation at early stages within $24 \mathrm{~h}$.

In the present study involving CS model rats, we hypothesized that treatment with icing will inhibit the influence of potassium by vasoconstriction, exert anti-inflammatory effects in the affected myocytes, and improve mitochondrial function.

\section{Materials and methods}

Animal model of CS. Male Wistar rats, weighing 250-300 g each, were obtained from Japan SLC (Shizuoka, Japan) and housed in a room that was maintained at a temperature of $23 \pm 3^{\circ} \mathrm{C}$ and relative humidity of $55 \pm 15 \%$ with $12: 12$-h light/dark cycle, with free access to food and water. The animal experiments were carried out according to the guidelines for animal use and were approved by the Life Science Research Center of Josai University (approval no. H29030). In the experimental periods, rats were anesthetized using sodium pentobarbital (50 mg/kg body weight, intraperitoneal [i.p.] administration). Anesthesia was maintained (confirmed by normal rectal temperature, respiratory rate, and sleeping state) by the i.p. administration of sodium pentobarbital $(20 \mathrm{mg} / \mathrm{kg}$ body weight/h). The body temperature was maintained uniformly throughout the experiment using a heating pad. The CS rat model has been previously described in detail (5). Briefly, a rubber tourniquet was applied to both the hindlimbs of each rat, which was wrapped five times around a $2.0 \mathrm{~kg}$-metal cylinder, and the end of the band was glued. After compression for $5 \mathrm{~h}$, it was released by cutting the band and removing the rubber tourniquet (i.e., reperfusion $0 \mathrm{~h}$ ).

Experimental design. Experimental design-1 (survival rate): To examine the survival period and the changes in the blood pressure and blood gas parameters of CS rats, they were randomly divided into the following six groups: i) Sham $(\mathrm{n}=10)$; ii) CS $(\mathrm{n}=10)$; iii) icing for 30 min over the entire hindlimb (CI-30)(n=10); iv) icing for 180 min over the entire hindlimb (CI-180)(n=10); v) local icing for 30 min over the hindlimb (CLI-30)(n=10); and (vi) local icing for $180 \mathrm{~min}$ over the hindlimb (CLI-180) $(n=10)$. All the rats used in the experiments were euthanized (confirmation by pupillary reflex to light) by sodium pentobarbital overdose $(100 \mathrm{mg} / \mathrm{kg}$ body weight, intravenous administration) at $48 \mathrm{~h}$ after all measurements were recorded.

Experimental design-2 (vital signs and blood gas parameters): To examine the changes in the blood pressure and blood gas parameters, the rats were randomly divided into the following six groups ( $\mathrm{n}=4$ each):i) sham; ii) CS; iii) CI-30; iv) CI-180; v) CL-30; and (vi) CLI-180 groups. Sequential sampling was performed at $0.083,0.5,1,3,6$, and $24 \mathrm{~h}$ after reperfusion.

Experimental design-3 (blood perfusion in the crushed hindlimb): blood perfusion in the crushed hindlimb was evaluated with the rats divided into the following four experimental groups ( $\mathrm{n}=6$ each): i) sham; ii) CS; iii) CI-30; and iv) CLI-30. Sequential measuring was performed at $0.083,0.5,1,3,6$ and $24 \mathrm{~h}$ after reperfusion. All the rats used in the experiments were euthanized (confirmation by pupillary reflex to light) by sodium pentobarbital overdose $(100 \mathrm{mg} / \mathrm{kg}$ body weight, intravenous administration) at $24 \mathrm{~h}$ after all measurements were recorded.

Experimental design-4 (plasma potassium $\left(\mathrm{K}^{+}\right)$and creatine phosphokinase (CPK) levels, and anti-inflammatory and mitochondrial function parameters): Biochemical analyses, serum cytokine levels, and mitochondrial parameters were evaluated with the rats divided into the following four experimental groups ( $\mathrm{n}=6$ each): i) Sham; ii) CS; iii) CLI-30; and (iv) CLI-180. Samplings were performed at 1, 3, 6 and $24 \mathrm{~h}$ after reperfusion. All the rats used in the experiments were euthanized (confirmation by pupillary reflex to light) by sodium pentobarbital overdose $(100 \mathrm{mg} / \mathrm{kg}$ body weight, intravenous administration) at each sampling time.

Vital signs, blood gas levels, and biochemical parameters. The following vital signs were recorded using a PowerLab data acquisition system (AD Instruments, Nagoya, Japan): Mean blood pressure (MBP), heart rate (HR), and body temperature (BT). One carotid artery was cannulated with a polyethylene catheter (PE-50 tubing) and was connected to a pressure transducer. Arterial blood samples from each group were obtained at $0.083,0.5,1,3,6,12$, and $24 \mathrm{~h}$ after reperfusion using the carotid artery catheter (6). The arterial levels of hematocrit (Hct), $\mathrm{pH}$, base excess (BE), anion gap (AG), and lactate were analyzed using i-STAT300F blood gas analyzer and CG4+ and EC8+ cartridges (FUSO Pharmaceutical Industries, Osaka, Japan). These measurements were assess using experimental design-2.

Venous blood from the jugular vein was collected and centrifuged to measure plasma $\mathrm{K}^{+}$and $\mathrm{CPK}$ (the measurements were carried out by SRL Inc., Tokyo, Japan). These measurements were performed using experimental design-3.

Blood perfusion parameters. Blood perfusion (BPF) of muscles and blood vessels was measured using PeriScan PIM3 (PERIMED, Stockholm, Sweden) in experimental design-3. BPF was assessed under the following conditions: distance to hindlimb: $10-15 \mathrm{~cm}$; measurement area: $10 \times 10 \mathrm{~cm}$; environmental temperature: $23 \pm 5^{\circ} \mathrm{C}$; and relative humidity: $50 \pm 5 \%$.

Determination of cytokines, reactive oxygen species (ROS) production, and mitochondrial function. The serum high 
mobility group box 1 (HMGB1), interleukin-6 (IL-6), and interleukin-10 (IL-10) were measured using HMGB1 ELISA Kit II (Shino-Test Co., Tokyo, Japan), Rat IL-6 Quantikine ${ }^{\circledR}$ ELISA kit (R\&D Systems, Inc., MN, USA), and Rat IL-10 Quantikine ${ }^{\circledR}$ ELISA kit (R\&D Systems, Inc., MN, USA) according to the manufacturer's instructions. ROS production in the injured gastrocnemius muscle was determined by measuring the concentration of thiobarbituric acid reactive substances (TBARS) (6). Myeloperoxidase (MPO) activity was determined by the method previously described by Murata et al (6). Isolation of mitochondria from the injured muscles for evaluation of mitochondrial function was performed using a mitochondrial isolation kit (Thermo Fisher Scientific K.K., Kanagawa, Japan). In mitochondrial permeability transition, mitochondrial membrane potential (i.e., mitochondrial inner membrane function) was evaluated using JC-1 Mitochondrial Membrane Potential Assay Kit (Cayman Chemical Company, Ann Arbor, MI, USA). To evaluate the mitochondrial outer membrane function, cytochrome c (cyt c) in the cytoplasm of the crushed myocytes from the samples that did not include mitochondria was quantified using Quantikine ${ }^{\circledR}$ cyt c Immunoassay (R\&D Systems, Inc. MN, USA). These measurements were performed using experimental design-4 with samples collected from the hindlimb muscles at 1, 3, 6 and $24 \mathrm{~h}$ after reperfusion.

Statistical analyses. Results are expressed as means \pm standard error of mean (SEM). Differences between the groups were assessed by analysis of variance with Tukey's honest significant difference test or Tukey's test. Survival curves were generated using the Kaplan-Meier method, and survival was compared by the log-rank test. $\mathrm{P}<0.05$ was considered to indicate a statistically significant difference (Statcel 2, 2nd ed. OMS Publishing Inc., Saitama, Japan).

\section{Results}

The impact of icing on CS rat viability. The survival rate is illustrated in Fig. 1. The survival rates of rats in the CS group were $91 \%, 82 \%, 36 \%, 13 \%$, and $13 \%$ at 1, 3, 6, 24, and $48 \mathrm{~h}$ after reperfusion, respectively. Until $6 \mathrm{~h}$ after reperfusion, the survival rates in CI-30, CI-180, CLI-30, and CLI-180 groups were significantly higher than those in the CS group. Until $24 \mathrm{~h}$ after reperfusion, the survival rates in the CI-30 and CLI-30 groups were significantly higher than those in the CS group; however, $48 \mathrm{~h}$ after reperfusion, the survival rates were not significantly different. Furthermore, icing for $180 \mathrm{~min}$ (CI-180 and CLI-180 groups) demonstrated a tendency to extend the survival period when compared with an icing period of $30 \mathrm{~min}$ (CI-30 and CLI-30 groups). Furthermore, the survival rates following icing for 5 and 60 min were similar to that following an icing period of $30 \mathrm{~min}$ (data not shown).

Icing treatment improves vital signs. The relationship between BT and lactate levels, and MBP and HR is illustrated in Fig. 2. BT in the CS group was significantly lower than that in the sham group at $24 \mathrm{~h}$ after reperfusion $\left(31 \pm 1.4\right.$ vs. $36 \pm 0.5^{\circ} \mathrm{C}$, $\mathrm{P}<0.05)$, and the lactate level in the CS group was significantly higher than that in the sham group throughout the experimental period. In the CI-30 and CLI-30 groups, BT levels

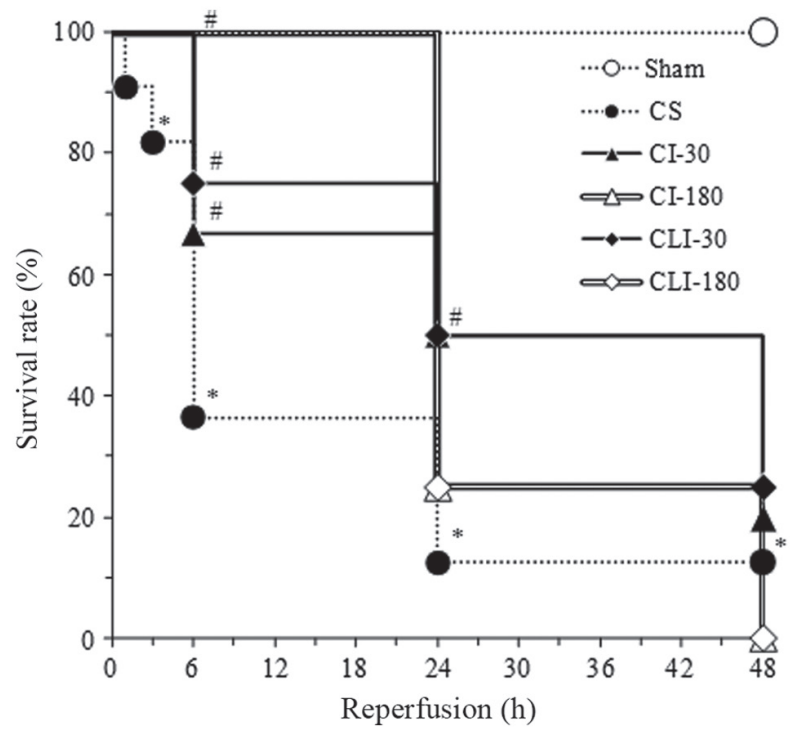

Figure 1. The impact of icing of varying durations on CS rat viability. Open circle: Sham group; closed circle: CS group; closed triangle: CI-30 group; open triangle: CI-180 group; closed diamond: CLI-30 group; and open diamond: CLI-180 group. *; vs. sham group $(\mathrm{P}<0.05)$, \#; vs. CS group $(\mathrm{P}<0.05)$ (log-rank test). CS, crush syndrome; CI, icing over the entire hindlimb; CLI, local icing.

were not significantly different when compared with that in the CS group until $6 \mathrm{~h}$ after reperfusion, and similarly than the sham group $\left(37 \pm 0.2\right.$ and $\left.36 \pm 0.4^{\circ} \mathrm{C}\right)$, and lactate levels were not showed significantly changes compared that the CS group. In the CI-180 and CLI-180 groups, the BT levels were significantly lower than that in the CS groups at $3 \mathrm{~h}$ after reperfusion $\left(27 \pm 0.9\right.$ and $28 \pm 2.3$ vs. $\left.35 \pm 0.5^{\circ} \mathrm{C}, \mathrm{P}<0.05\right)$, and the lactate levels were significantly higher $(7.6 \pm 0.9$ and $6.9 \pm 1.0 \mathrm{vs}$. $4.5 \pm 0.4 \mathrm{mmol} / \mathrm{l}, \mathrm{P}<0.05)$. In the CS group, MBP and HR levels were significantly lower than that in the sham group until $24 \mathrm{~h}$ after reperfusion (MBP, $42.8 \pm 3.8$ vs. $123.2 \pm 3.1 \mathrm{mmHg}$; HR, $231 \pm 20$ vs. $390 \pm 64$ bpm; $\mathrm{P}<0.05$ at $24 \mathrm{~h}$ after reperfusion). In the CI-30, CLI-30, CI-180, and CLI-180 groups, MBP levels were significantly higher than that in the CS group until $24 \mathrm{~h}$

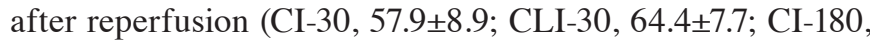
73.3 \pm 4.1 ; CLI-180, 67.2 $\pm 4.2 \mathrm{mmHg}$ ), and the HR levels were the CI-30 and CLI-30 group has similarly than the sham group until $24 \mathrm{~h}$ after reperfusion (CI-30, 362 \pm 25 ; CLI-30, $352 \pm 7 \mathrm{bpm}$ ) however that the CI-180 and CLI-180 group were similar in the CS group at $6 \mathrm{~h}$ after reperfusion (CI-180, $293 \pm 18$; CLI-180, 322 \pm 9 bpm) and significantly decreased compared to the CI-30 and CLI-30 groups.

Blood gas parameters and Hct levels of all the experimental groups are presented in Table I. $\mathrm{pH}$ and $\mathrm{BE}$ levels in the CS groups were significantly lower than that in the sham group, while the Hct level was significantly higher than that in the sham group. In the CI-30 and CLI-30 groups, $\mathrm{pH}$ level significantly increased compared to the CS group at 6 and $24 \mathrm{~h}$ after reperfusion; however, the CI-180 and CLI-180 groups had worse outcomes compared with the CS group until 0.083 to $1 \mathrm{~h}$ after reperfusion.

Icing treatment attenuated hyperkalemia by controlling BPF. The survival rates, vital signs, and blood gas parameters 

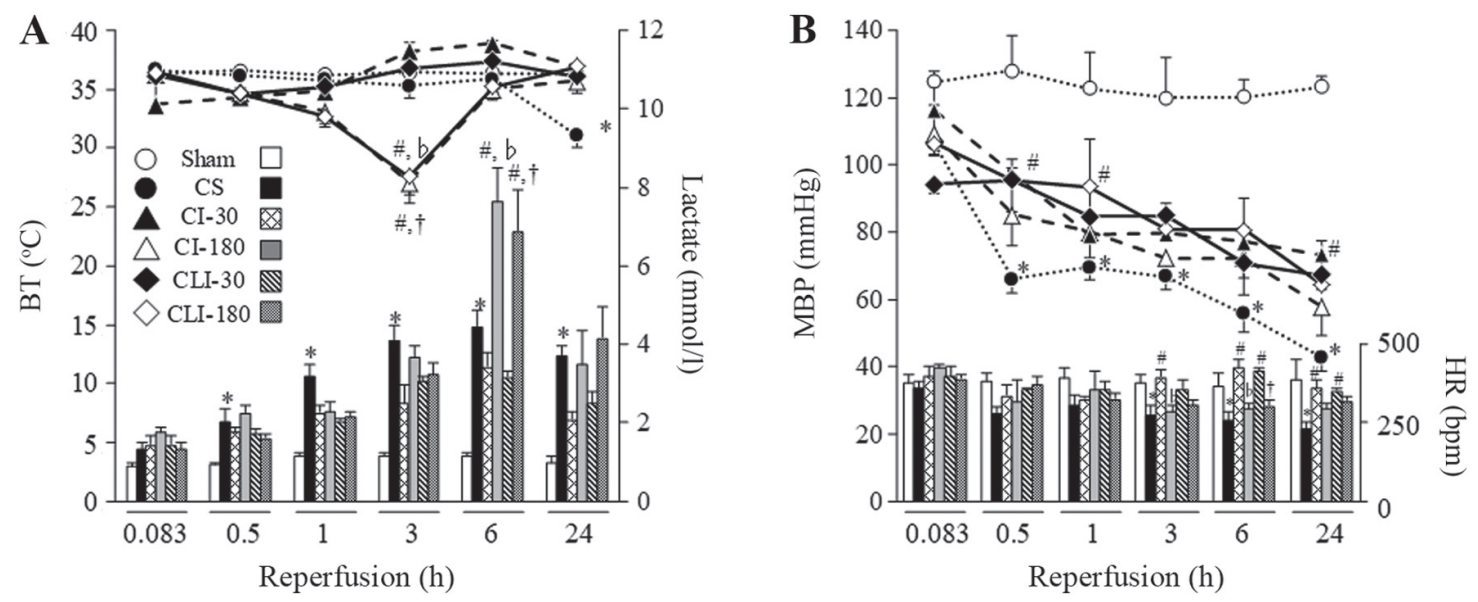

Figure 2. Icing treatment improves vital signs. a) The relationship between BT and lactate levels, (B) the relationship between MBP and HR levels. The line graph is compares BT and MBP, while the bar graph lactate and HR levels. Open circle and white bar: Sham group; closed circle and black bar: CS group; closed triangle and shaded bar: CI-30 group; open triangle and gray bar: CI-180 group; closed diamond and diagonal bar: CLI-30 group; and open diamond and black dot bar: CLI-180 group. Values are showed as mean \pm standard error of mean, ${ }^{*}$ vs. sham group $(\mathrm{P}<0.05) ;{ }^{*}$ vs. CS group $(\mathrm{P}<0.05) ; \dagger$ vs. CI-30 group $(\mathrm{P}<0.05)$; and 'vs. CLI-30 group $(\mathrm{P}<0.05)$ (Tukey's test). CS, crush syndrome; CI, icing on whole hindlimb of CS rat; CLI, icing on local hindlimb of CS rat; $\mathrm{BT}$, body temperature; MBP, mean blood pressure and HR, hart rate.

improved when the the icing period was over 30 min. Further, an icing period of $180 \mathrm{~min}$ induces hypothermia; therefore, we decided that the icing period should be $30 \mathrm{~min}$.

The plasma $\mathrm{K}^{+}$and CPK levels and BPF of the crushed limbs is presented in Fig. 3. CS group had significantly higher plasma $\mathrm{K}^{+}$and CPK levels than the sham group at all experimental periods, while the same in the CI-30 and CLI-30 groups were significantly lower than that in the CS group at $24 \mathrm{~h}$ after reperfusion. In the CS group, BPF level within the blood vessels was not significantly different from that in the sham group, and the muscle BPF level was significantly lower than that in the sham group at all experimental periods. In the CI-30 and CLI-30 groups, BPF within the blood vessels was significantly lower than that in the CS group at 0.5 and $1 \mathrm{~h}$ after reperfusion, while the muscle BPF level was similar to that in the CS group between 0.5-6 $\mathrm{h}$ after reperfusion.

Icing treatment attenuated inflammation through prevention of mitochondrial dysfunction. The serum cytokine levels, and TBARS (i.e., ROS generation level) due to mitochondrial dysfunction is presented in Figs. 4 and 5. The CS group had significantly higher serum IL- 6 and IL-10 levels than the sham group at 3,6 , and $24 \mathrm{~h}$ after reperfusion, and showed maximum levels at $6 \mathrm{~h}$ after reperfusion. Serum HMGB1 and MPO levels in the CS group were significantly higher than that in the sham group at 3-24 $\mathrm{h}$ after reperfusion. Their levels in the CI-30 and CLI-30 groups were significantly lower than that in the CS group (Fig. 4). While evaluating the mitochondrial function, TBARS level in the CS group was maximum at $6 \mathrm{~h}$ after reperfusion and was significantly higher than that in the sham group at 3 and $6 \mathrm{~h}$ after reperfusion. Furthermore, JC-1 fluorescence (mitochondrial membrane potential) and mitochondrial cyt c content in the CS group was significantly lower than that in the sham group at all experimental timepoints, and cytoplasm cyt c content of the CS group was lower than that in the sham group. In the CI-30 and CLI-30 groups, TBARS levels were significantly lower than that in the CS group at 3 and $6 \mathrm{~h}$ after reperfusion, JC-1 fluorescence was significantly higher than that in the CS group at 6 and $24 \mathrm{~h}$ after reperfusion, and mitochondrial cytochrome $\mathrm{C}$ contents were significantly increased and cytoplasm cytochrome C contents tended to be decreased compared with those in the CS group (Fig. 5).

\section{Discussion}

A variety of modalities are used to treat muscle injuries including (7) cryotherapy, massage therapy, and hyperbaric oxygen therapy; however, icing is the mainstay treatment for several types of acute musculoskeletal injuries $(12,13)$.

We selected the icing method since icing periods of both 30 and $180 \mathrm{~min}$ decrease the average temperature of the skin $\left(32-28^{\circ} \mathrm{C}\right)$ by $3-5^{\circ} \mathrm{C}$ (data not shown). In this study, BT significantly decreased only with an icing period of $180 \mathrm{~min}$. Previous studies reported that ice application of $30 \mathrm{~min}$ over the Achilles (14) and patellar (15) tendons decreased the skin temperature to approximately $17-20^{\circ} \mathrm{C}$. Furthermore, Dewhurst et al (16) reported that ice pack application over the thigh decreases skin temperature by $6^{\circ} \mathrm{C}$ and muscle temperature by $4^{\circ} \mathrm{C}$. Similarly, in our study, the icing procedure was able to be cool the injured muscle. Knight et al (8), suggested that the effects of local cooling were limited to local effects; however, it appears to be not so according to this study. An icing period of $30 \mathrm{~min}$ did not decreased BT; however, an icing period of $180 \mathrm{~min}$ did significantly decrease BT. This might be because of additional factors such as increased lactate and decrease HR because the gastrocnemius is a relatively large muscle in the rat. Additionally, the vasoconstriction in the affected part induced by icing would decrease the release of potassium into the systemic circulation. We demonstrated that hyperkalemia can be improved from blocking the blood flow to the crushed limbs (data not shown). In the present study, we demonstrated that the blood flow to the affected limb temporarily decreases with icing treatment (Fig. 3D), and that these changes had similar effects on decreasing serum potassium in the experimental period (Fig. 3A). In contrast, 
Table I. Effects of CS and icing therapy on blood gas parameters.

\begin{tabular}{|c|c|c|c|c|c|c|}
\hline \multirow[b]{2}{*}{ Parameter } & \multicolumn{6}{|c|}{ Reperfusion (h) } \\
\hline & 0.083 & 0.5 & 0.1 & 3 & 6 & 24 \\
\hline \multicolumn{7}{|l|}{$\mathrm{pH}$} \\
\hline sham & $7.48 \pm 0.02$ & $7.46 \pm 0.01$ & $7.46 \pm 0.03$ & $7.48 \pm 0.00$ & $7.50 \pm 0.01$ & $7.45 \pm 0.01$ \\
\hline $\mathrm{CS}$ & $7.44 \pm 0.02$ & $7.51 \pm 0.03$ & $7.43 \pm 0.02$ & $7.38 \pm 0.02^{\mathrm{a}}$ & $7.31 \pm 0.02^{\mathrm{a}}$ & $7.22 \pm 0.05^{\mathrm{a}}$ \\
\hline CI-30 & $7.45 \pm 0.04$ & $7.42 \pm 0.03$ & $7.44 \pm 0.02$ & $7.43 \pm 0.03$ & $7.40 \pm 0.00^{\mathrm{b}}$ & $7.37 \pm 0.03^{b}$ \\
\hline CI- 180 & $7.42 \pm 0.01$ & $7.31 \pm 0.06^{\mathrm{b}}$ & $7.33 \pm 0.03^{\mathrm{b}}$ & $7.31 \pm 0.03$ & $7.29 \pm 0.03$ & $7.26 \pm 0.03$ \\
\hline CLI-30 & $7.42 \pm 0.03$ & $7.38 \pm 0.01$ & $7.38 \pm 0.02$ & $7.38 \pm 0.03$ & $7.40 \pm 0.04^{\mathrm{b}}$ & $7.37 \pm 0.03^{b}$ \\
\hline CLI-180 & $7.38 \pm 0.01^{\mathrm{b}}$ & $7.37 \pm 0.02^{\mathrm{b}}$ & $7.35 \pm 0.02^{\mathrm{b}}$ & $7.30 \pm 0.03$ & $7.27 \pm 0.03$ & $7.30 \pm 0.04$ \\
\hline \multicolumn{7}{|c|}{$\mathrm{BE}(\mathrm{mmol} / \mathrm{l})$} \\
\hline sham & $2.3 \pm 0.6$ & $2.3 \pm 1.0$ & $2.8 \pm 0.9$ & $3.0 \pm 1.1$ & $2.5 \pm 0.3$ & $2.8 \pm 1.0$ \\
\hline $\mathrm{CS}$ & $2.0 \pm 0.5$ & $2.3 \pm 0.7$ & $2.0 \pm 1.4$ & $0.0 \pm 2.1$ & $-5.0 \pm 0.7^{\mathrm{a}}$ & $-7.8 \pm 1.5^{\mathrm{a}}$ \\
\hline CI-30 & $0.5 \pm 0.7$ & $-2.3 \pm 1.0$ & $-3.3 \pm 1.7$ & $-5.0 \pm 2.0$ & $-7.5 \pm 1.4$ & $-6.3 \pm 1.2$ \\
\hline CI-180 & $0.5 \pm 0.6$ & $-2.0 \pm 1.2$ & $-1.5 \pm 1.1$ & $-5.5 \pm 1.4$ & $-6.0 \pm 0.8$ & $-6.5 \pm 1.1$ \\
\hline CLI-30 & $0.5 \pm 2.5$ & $0.0 \pm 1.6$ & $0.0 \pm 2.4$ & $-4.8 \pm 3.5$ & $-5.8 \pm 2.2$ & $-4.8 \pm 1.7$ \\
\hline CLI-180 & $-0.5 \pm 0.7$ & $-1.8 \pm 0.7$ & $-0.8 \pm 0.3$ & $-3.8 \pm 0.3$ & $-10.0 \pm 0.9^{b}$ & $-9.5 \pm 0.3$ \\
\hline \multicolumn{7}{|c|}{$\mathrm{AG}(\mathrm{mmol} / \mathrm{l})$} \\
\hline sham & $15.3 \pm 1.5$ & $13.0 \pm 1.6$ & $14.0 \pm 0.8$ & $13.0 \pm 1.7$ & $13.7 \pm 1.5$ & $15.0 \pm 1.2$ \\
\hline $\mathrm{CS}$ & $19.3 \pm 1.0$ & $15.5 \pm 1.4$ & $15.0 \pm 1.2$ & $15.8 \pm 1.6$ & $17.3 \pm 1.9$ & $19.6 \pm 1.0$ \\
\hline CI-30 & $16.0 \pm 2.3$ & $18.8 \pm 1.3$ & $18.5 \pm 1.5$ & $18.5 \pm 1.1$ & $19.0 \pm 0.7$ & $19.5 \pm 0.7$ \\
\hline CI-180 & $20.8 \pm 1.5$ & $18.5 \pm 0.6$ & $16.3 \pm 0.9$ & $18.5 \pm 1.4$ & $19.3 \pm 1.1$ & $18.8 \pm 0.6$ \\
\hline CLI-30 & $18.0 \pm 1.6$ & $15.8 \pm 1.8$ & $15.8 \pm 1.4$ & $17.8 \pm 1.2$ & $19.8 \pm 1.7$ & $21.0 \pm 1.7$ \\
\hline CLI-180 & $20.8 \pm 1.7$ & $16.8 \pm 1.1$ & $14.5 \pm 2.0$ & $16.5 \pm 1.7$ & $19.8 \pm 0.6$ & $21.5 \pm 1.7$ \\
\hline \multicolumn{7}{|l|}{ Hct $(\%)$} \\
\hline sham & $45.0 \pm 1.2$ & $46.8 \pm 1.3$ & $47.3 \pm 1.7$ & $46.5 \pm 1.4$ & $46.3 \pm 1.3$ & $46.0 \pm 1.7$ \\
\hline $\mathrm{CS}$ & $45.0 \pm 1.2$ & $46.5 \pm 0.3$ & $47.5 \pm 0.3$ & $52.0 \pm 1.2$ & $56.0 \pm 1.8^{\mathrm{a}}$ & $60.5 \pm 1.1^{\mathrm{a}}$ \\
\hline CI-30 & $46.5 \pm 1.7$ & $45.5 \pm 1.5$ & $45.8 \pm 2.2$ & $49.5 \pm 1.5$ & $52.3 \pm 2.2$ & $51.8 \pm 0.3$ \\
\hline CI-180 & $47.0 \pm 0.7$ & $48.3 \pm 0.7$ & $51.3 \pm 0.9$ & $52.8 \pm 0.7$ & $54.8 \pm 0.7$ & $53.8 \pm 1.2$ \\
\hline CLI-30 & $45.0 \pm 2.4$ & $47.0 \pm 2.4$ & $47.3 \pm 2.3$ & $51.3 \pm 2.8$ & $52.3 \pm 2.1$ & $51.3 \pm 1.7$ \\
\hline CLI-180 & $46.3 \pm 2.0$ & $50.0 \pm 0.8$ & $50.3 \pm 0.7$ & $53.5 \pm 1.1$ & $56.0 \pm 1.4$ & $55.3 \pm 1.4$ \\
\hline
\end{tabular}

Values are presented as mean \pm standard error in mean; ${ }^{\mathrm{a}} \mathrm{vs}$. sham group and ${ }^{\mathrm{b}} \mathrm{vs}$. CS group $(\mathrm{P}<0.05)$ (Tukey's test). CS, crush syndrome; CI, icing over the entire hindlimb of CS rats; CLI, local icing over the affected hindlimb of CS rats; BE, base excess; AG, anion gap; Hct, hematocrit.

Nakayama et al (17) reported that warming of the hindlimbs to $40^{\circ} \mathrm{C}$ during compression resulted in a much lower survival rate and significantly enhanced hyperkalemia, leading to cardiac arrest. Therefore, lower BT and temperature of the affected muscle are believed to be necessary in improving hyperkalemia. However, icing treatment for $30 \mathrm{~min}$ significantly increased the survival rate within $24 \mathrm{~h}$ from the injury; however, that with icing treatment for $180 \mathrm{~min}$ did not vary significantly despite a temporary decrease in serum potassium. The CS model rat in our study died from cardiac insufficiency following rapidly progressing (within an injured $3 \mathrm{~h}$ ) hyperkalemia (5); nevertheless, the decreased survival period following hypothermia by the icing treatment for $180 \mathrm{~min}$ could not provide this reason. We believe that survival with CS state may be affected by various factors, such as hypovolemic shock and inflammation. Therefore, our findings suggest that icing therapy for $30 \mathrm{~min}$ effectively prolonged the viability in situations where the risk of hypothermia could be controlled, although, cooling of the affected limb is a simple therapy in difficult clinical situations with ongoing CS.

Interestingly, low tissue temperature can help in resisting hypoxia because chemical reactions in vivo are catalyzed by enzymes (18). Furthermore, this anti-inflammation effect prevents leukocyte migration and edema by reducing vascular permeability (19). Icing treatment is not considered to be beneficial in the repair process of damaged tissue $(8,20,21)$; however, uncontrolled inflammation is also harmful for homeostasis. We evaluated the overall balance between 

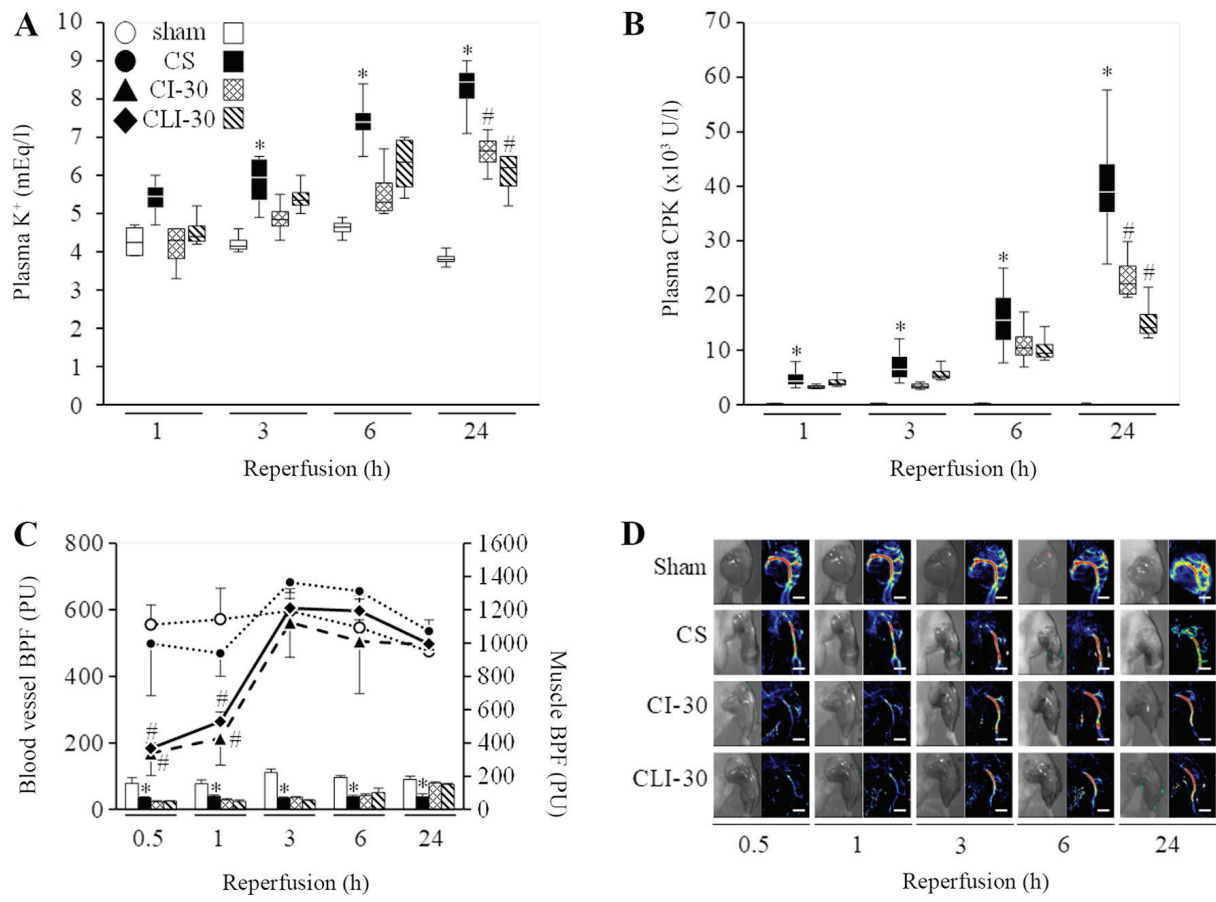

Figure 3. Attenuation of hyperkalemia secondary to icing treatments. (A) plasma $\mathrm{K}^{+}$level, (B) plasma CPK level, (C) the relationship between blood vessel BPF and muscle BPF levels and (D) laser doppler image showing the changes in an affected hindlimb. Scale bar=1 cm. White bar: Sham group; black bar: CS group; shaded bar: CI-30 group; and diagonal bar: CLI-30 group. Values are presented as mean \pm standard error in mean; "vs. sham group (P<0.05); "vs. CS group $(\mathrm{P}<0.05)$ (Tukey's test). CS, crush syndrome; $\mathrm{CI}$, icing over the entire hindlimb of CS rats; CLI, local icing over the hindlimb of CS rats; K+, potassium; CPK, creatine phosphokinase; BPF, blood perfusion.
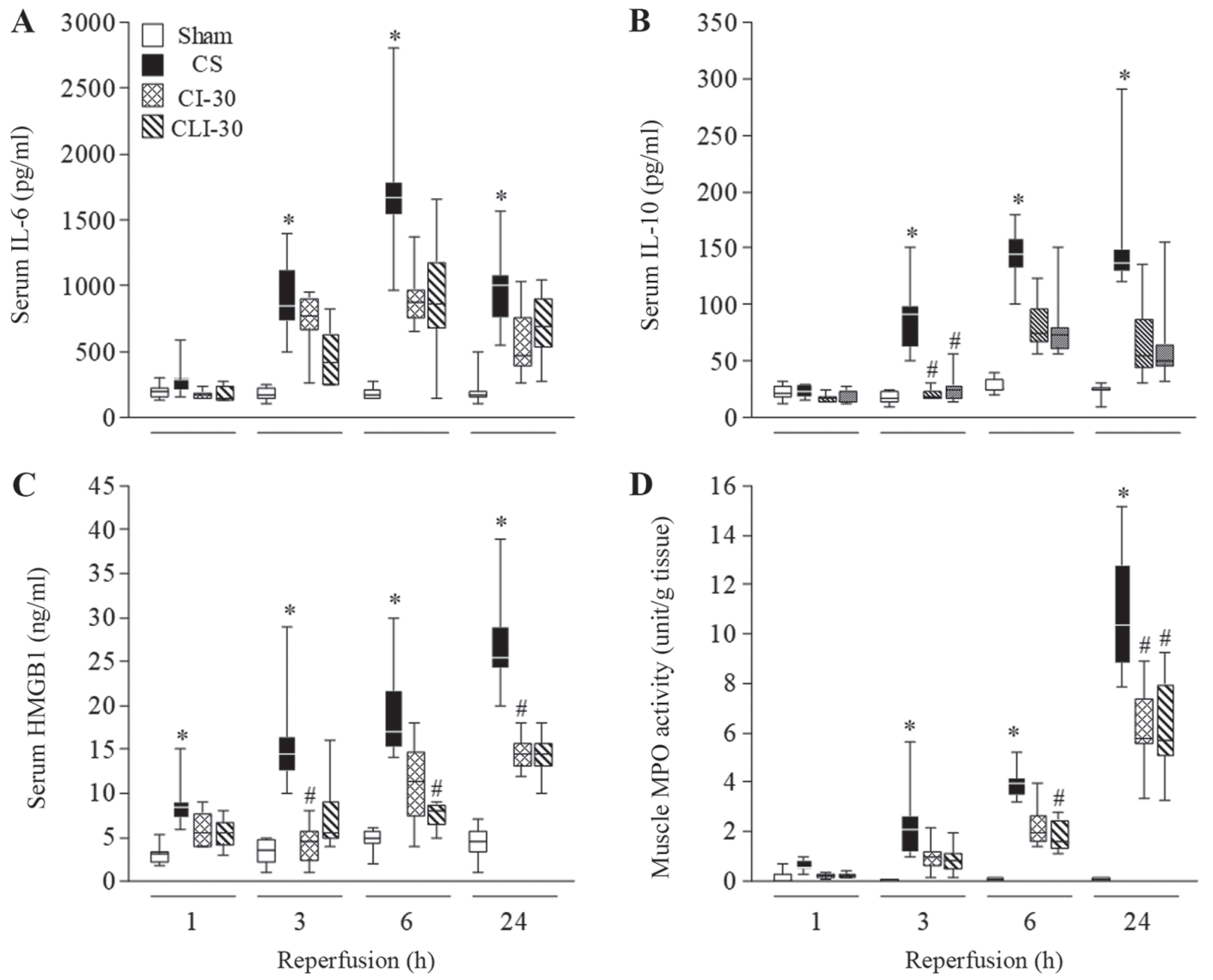

Figure 4. Icing treatment was attenuated inflammation. (A) serum IL-6, (B) serum IL-10, (C) serum HMGB1 and (D) muscle tissue MPO activity. White bar: Sham group; black bar: CS group; shaded bar: CI-30 group; and diagonal bar: CLI-30 group. Values are showed as mean \pm standard error in mean; *vs. sham group $(\mathrm{P}<0.05)$; ${ }^{*}$ vs. CS group $(\mathrm{P}<0.05)$ (Tukey's test). CS, crush syndrome; CI, icing over the entire hindlimb of CS rats; CLI, local icing of hindlimb of CS rats; IL, interleukin; HMGB1, high mobility group box protein 1; MPO, myeloperoxidase. 

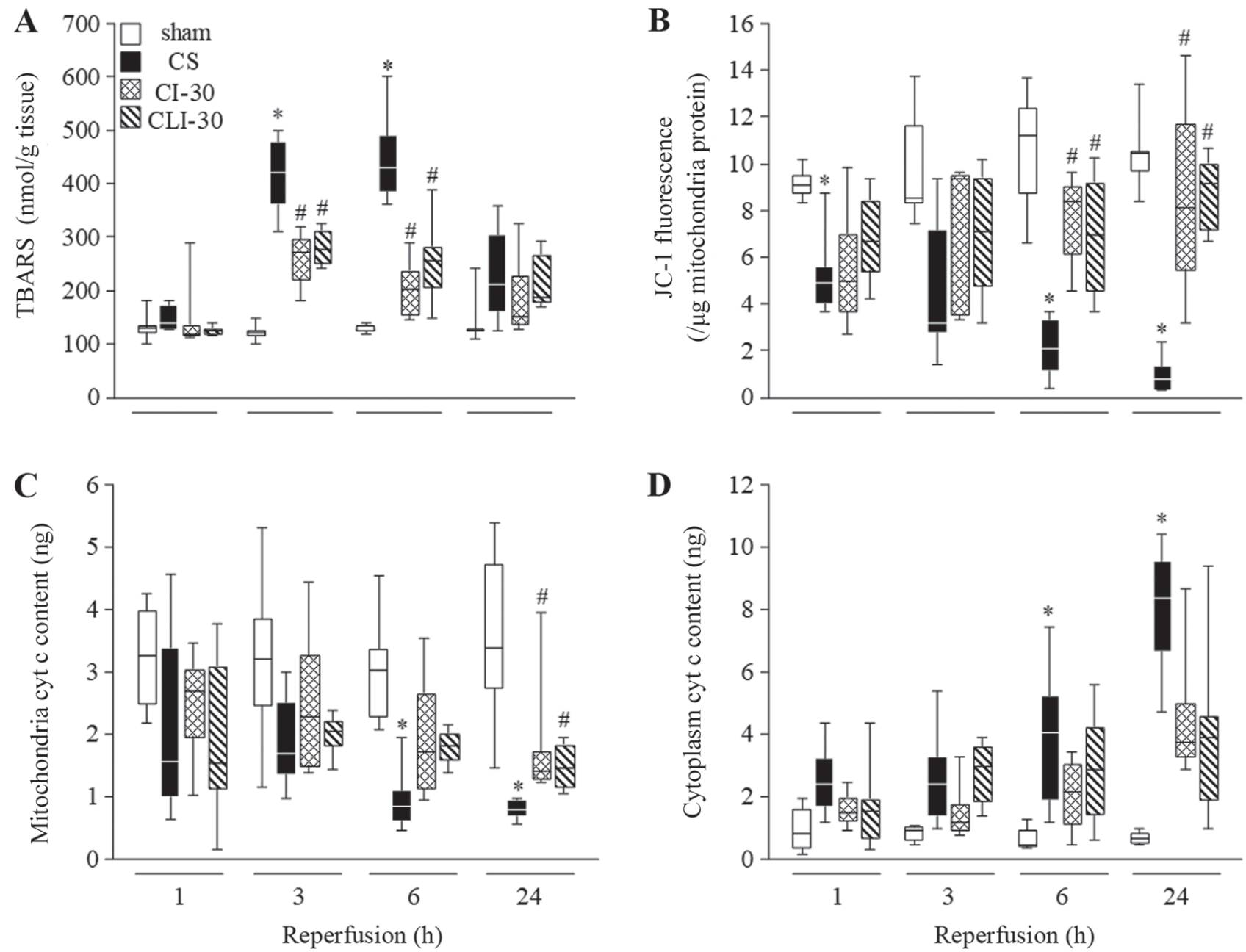

Figure 5. Improving ROS generation and prevention of mitochondrial dysfunction in the crushed limb. (A) TBARS level, (B) JC-1 fluorescence, reflecting mitochondria membrane potential, (C) mitochondria cyt c content level and (D) cytoplasm cyt c content level in the crushed muscle tissue. White bar: Sham group; black bar: CS group; shaded bar: CI-30 group; and diagonal bar: CLI-30 group. Values are showed as mean \pm standard error in mean; "vs. sham group $(\mathrm{P}<0.05)$ and "vs. CS group $(\mathrm{P}<0.05)$ (Tukey's test). CS, crush syndrome; CI, icing over the entire hindlimb of CS rats; CLI, local icing over the hindlimb of CS rats; TBARS, thiobarbituric acid reactive substance; cyt c, cytochrome $c$.

inflammatory and anti-inflammatory factors by assessing the serum levels of cytokines IL-6 and IL-10 (22), which are markedly induced in CS rats. Additionally, we evaluated the serum levels of HMGB1, which is lethally involved in constitutive expression and vascular endothelial cell interactions $(23,24)$, and is a lethal inflammation mediator (25). Extracellular release of HMGB1 induces the expression of adhesion factors of intravascular cells such as vascular cell adhesion molecule 1, intercellular adhesion molecule 1, and E-selectin, and inflammatory reactions involving production of cytokines due to the extravasation of neutrophils and monocytes $(23,26)$. As demonstrated by the MPO activity level in the muscle tissue, icing treatment suppresses both neutrophil infiltration and HMGB1 expression. The reduction in HMGB1, in turn, suppresses inflammation as indicated by lowered levels of IL-6 and IL-10, which can also be due to inhibition of cell rupture. However, although IL-10 is an anti-inflammation cytokine, lowered levels of it seemed to be secondary to no expression due to suppression of inflammation. Additionally, icing treatment affects mitochondrial function after a long period of time. One of the characteristics of CS is that ROS production is enhanced in a relatively short time along with decreased mitochondrial function (6). In this study, ROS production peaked $6 \mathrm{~h}$ after the compression injury and was reduced by icing treatment. It is believed that this result is because of normalization of mitochondrial function and mitochondrial injury secondary to decreased cell temperature, as demonstrated by maintenance of the mitochondrial membrane potential (Fig. 5B) as well as cytoplasmic of cyt c (Figs. 5C and D). Therapeutic hypothermia may also alleviate bursts of reactive oxygen species and calcium overload and activate the cell survival signaling pathways (26-30), which are believed to be involved in permeability transition pore (PTP) regulation (31-33). Furthermore, some reports have suggested that therapeutic hypothermia limits calcium-induced mitochondrial dysfunction, including opening of PTP $(26,34,35)$.

In emergency situations, icing treatment following crush injury temporarily prolonged the viability by suppressing potassium elevation; however, this could not improve the overall outcomes dramatically because CS involves simultaneous and rapid worsening of multiple symptoms. Icing was suggested to be effective in suppressing the inflammatory reactions; therefore, the effectiveness of icing therapy can be enhanced by combining it with other infusion therapies. 


\section{Acknowledgments}

The authors thank Dr. Hiroyuki Uchida and Dr. Junta Ito for advice, and Mr. Ryo Kawanishi and Ms. Chikako Murata for technical assistance. These members were affiliated Laboratory of Drug Safety Management or Division of Pathophysiology, Department of Clinical Dietetics and Human Nutrition, Faculty of Pharmacy and Pharmaceutical Science, Josai University.

\section{Funding}

The present study was supported by JSPS KAKENHI (grant no. 16K20397).

\section{Availability of data and material}

The datasets used and/or analyzed during the current study are available from the corresponding author on reasonable request.

\section{Authors' contributions}

IM led the project, and designed and performed most of the experiments. MI and MK assisted with the survival and biochemical marker analyses. JK, YI, and IK conceived the study, participated in its design and coordination, and helped draft the manuscript. All authors have read and approved the final manuscript.

\section{Ethics approval and consent to participate}

The present study was approved by the Life Science Research Center of Josai University (approval no. H29030).

\section{Patient consent for publication}

Not applicable.

\section{Competing interests}

The all authors declare that they have no competing interests.

\section{References}

1. Smith J and Greaves I: Crush injury and crush syndrome: A review. J Trauma 54 (5 Suppl): S226-S230, 2003.

2. Sever MS, Vanholder R and RDRTF of ISN Work Group on Recommendations for the Management of Crush Victims in Mass Disasters: Recommendation for the management of crush victims in mass disasters. Nephrol Dial Transplant 27 (Suppl 1): i1-i67, 2012

3. Gonzalez D: Crush syndrome. Crit Care Med 33 (1 Suppl): S34-S41, 2005.

4. Ashkenazi I, Isakovich B, Kluger Y, Alfici R, Kessel B and Better OS: Prehospital management of earthquake casualties buried under rubble. Prehosp Disaster Med 20: 122-133, 2005.

5. Murata I, Ooi K, Sasaki H, Kimura S, Ohtake K, Ueda H, Uchida H, Yasui N, Tsutsui Y, Yoshizawa N, et al: Characterization of systemic and histologic injury after crush syndrome and intervals of reperfusion in a small animal model. J Trauma 70: 1453-1463, 2011.

6. Murata I, Abe Y, Yaginuma Y, Yodo K, Kamakari Y, Miyazaki Y, Baba D, Shinoda Y, Iwasaki T, Takahashi K, et al: Astragaloside-IV prevents acute kidney injury and inflammation by normalizing muscular mitochondrial function associated with a nitric oxide protective mechanism in crush syndrome rats. Ann Intensive Care 7: 90, 2017.
7. Tiidus PM: Alternative treatments for muscle injury: Massage, cryotherapy, and hyperbaric oxygen. Curr Rev Musculoskelet Med 8: 162-167, 2015.

8. Merrick MA, Rankin JM, Andres FA, Hinman CL: A preliminary examination of cryotherapy and secondary injury in skeletal muscle. Med Sci Sports Exerc 31: 1516-1521, 1999.

9. Jahandiez V, Cour M, Bochaton T, Abrial M, Loufouat J, Gharib A, Varennes A, Ovize M and Argaud L: Fast therapeutic hypothermia prevents post-cardiac arrest syndrome through cyclophilin D-mediated mitochondrial permeability transition inhibition. Basic Res Cardiol 112: 35, 2017.

10. Singh DP, Barani Lonbani Z, Woodruff MA, Parker TJ, Steck R and Peake JM: Effects of topical icing on inflammation, angiogenesis, revascularization, and myofiber regeneration in skeletal muscle following contusion injury. Front Physiol 8: 93, 2017.

11. Takagi R, Fujita N, Arakawa T, Kawada S, Ishii N and Miki A: Influence of icing on muscle regeneration after crush injury to skeletal muscles in rats. J Appl Physiol (1985) 110: 382-388, 2011.

12. Meeusen R and Lievens P: The use of cryotherapy in sports injuries. Sports Med 3: 398-414, 1986.

13. Swenson C, Swärd L and Karlsson J: Cryotherapy in sports medicine. Scand J Med Sci Sports 6: 193-200, 1996.

14. Alegre LM, Hasler M, Wenger S, Nachbauer W and Csapo R: Does knee joint cooling change in vivo patellar tendon mechanical properties? Eur J Appl Physiol 116: 1921-1929, 2016.

15. Warren TA, McCarty EC, Richardson AL, Michener T and Spindler KP: Intra-articular knee temperature changes: Ice versus cryotherapy device. Am J Sports Med 32: 441-445, 2004.

16. Dewhurst S, Macaluso A, Gizzi L, Felici F, Farina D and De Vito G: Effects of altered muscle temperature on neuromuscular properties in young and older women. Eur J Appl Physiol 108: 451-458, 2010.

17. Nakayama T, Fujita M, Ishihara M, Ishihara M, Ogata S, Yamamoto Y, Shimizu M, Maehara T, Kanatani Y and Tachibana S: Improved survival rate by temperature control at compression sites in rat model of crush syndrome. J Surg Res 188: 250-259, 2014.

18. Merrick MA: Secondary injury after musculoskeletal trauma: A review and update. J Athl Train 37: 209-217, 2002.

19. Deal DN, Tipton J, Rosencrance E, Curl WW and Smith TL: Ice reduces edema. A study of microvascular permeability in rats. J Bone Joint Surg Am 84: 1573-1578, 2002.

20. Tidball JG and Wehling-Henricks M: Damage and inflammation in muscular dystrophy: Potential implications and relationships with autoimmune myositis. Autoimmune myositis. Curr Opin Rheumatol 17: 707-713, 2005.

21. Barnett A: Using recovery modalities between training sessions in elite athletes: Does it help? Sports Med 36: 781-796, 2006.

22. Murata I, Ooi K, Shoji S, Motohashi Y, Kan M, Ohtake K, Kimura S, Ueda H, Nakano G, Sonoda K, et al: Acute lethal crush-injured rats can be successfully rescued by a single injection of high-dose dexamethasone through a pathway involving PI3K-Akt-eNOS signaling. J Trauma Acute Care Surg 75: 241-249, 2013.

23. Scaffidi P, Misteli T and Bianchi ME: Release of chromatin protein HMGB1 by necrotic cells triggers inflammation. Nature 418: 191-195, 2002.

24. Lotze MT, and Tracey KJ: High-mobility group box 1 protein (HMGB1): Nuclear weapon in the immune arsenal. Nat Rev Immunol 5: 331-342, 2005.

25. Wang H, Bloom O, Zhang M, Vishnubhakat JM, Ombrellino M, Che J, Frazier A, Yang H, Ivanova S, Borovikova L, et al: HMG-1 as a late mediator of endotoxin lethality in mice. Science 285: 248-251, 1999.

26. Huang CH, Tsai MS, Chiang CY, Su YJ, Wang TD, Chang WT, Chen HW and Chen WJ: Activation of mitochondrial STAT-3 and reduced mitochondria damage during hypothermia treatment for post-cardiac arrest myocardial dysfunction. Basic Res Cardiol 110: 59, 2015.

27. Tissier R, Chenoune M, Pons S, Zini R, Darbera L, Lidouren F, Ghaleh B, Berdeaux A and Morin D: Mild hypothermia reduces per-ischemic reactive oxygen species production and preserves mitochondrial respiratory complexes. Resuscitation 84: 249-255, 2013.

28. Yang X, Liu Y, Yang XM, Hu F, Cui L, Swingle MR, Honkanen RE, Soltani P, Tissier R, Cohen MV and Downey JM: Cardioprotection by mild hypothermia during ischemia involves preservation of ERK activity. Basic Res Cardiol 106: 421-430, 2011.

29. Yenari MA and Han HS: Neuroprotective mechanisms of hypothermia in brain ischemia. Nat Rev Neurosci 13: 267-278, 2012.

30. Zhao H, Shimohata T, Wang JQ, Sun G, Schaal DW, Sapolsky RM and Steinberg GK: Akt contributes to neuroprotection by hypothermia against cerebral ischemia in rats. J Neurosci 25: 9794-9806, 2005. 
31. Alam MR, Baetz D and Ovize M: Cyclophilin D and myocardial ischemia-reperfusion injury: A fresh perspective. J Mol Cell Cardiol 78: 80-89, 2015.

32. Baines CP, Kaiser RA, Purcell NH, Blair NS, Osinska H, Hambleton MA, Brunskill EW, Sayen MR, Gottlieb RA, Dorn GW, et al: Loss of cyclophilin D reveals a critical role for mitochondrial permeability transition in cell death. Nature 434: 658-662, 2005

33. Bochaton T, Crola-Da-Silva C, Pillot B, Villedieu C, Ferrera L, Alam MR, Thibault H, Strina M, Gharib A, Ovize M and Baetz D: Inhibition of myocardial reperfusion injury by ischemic postconditioning requires sirtuin 3-mediated deacetylation of cyclophilin D. J Mol Cell Cardiol 84: 61-69, 2015.
34. Liu J, Wang Y, Zhuang Q, Chen M, Wang Y, Hou L and Han F. Protective effects of cyclosporine A and hypothermia on neuronal mitochondria in a rat asphyxial cardiac arrest model. Am J Emerg Med 34: 1080-1085, 2016.

35. Patil KD, Halperin HR and Becker LB: Cardiac arrest: Resuscitation and reperfusion. Circ Res 116: 2041-2049, 2015. 